ÉGYPTE monde arabe

\section{Égypte/Monde arabe}

12-13 | 1993

Une économie en transition

\title{
Les spécificités de l'économie égyptienne
}

\section{Bruno Cabrillac}

\section{(2) OpenEdition}

\section{Journals}

\section{Édition électronique}

URL : https://journals.openedition.org/ema/1240

DOI : 10.4000/ema.1240

ISSN : 2090-7273

\section{Éditeur}

CEDEJ - Centre d'études et de documentation économiques juridiques et sociales

\section{Édition imprimée}

Date de publication : 31 mars 1993

Pagination : 12-19

ISSN : 1110-5097

\section{Référence électronique}

Bruno Cabrillac, «Les spécificités de l'économie égyptienne », Égypte/Monde arabe [En ligne], 12-13 |

1993, mis en ligne le 08 juillet 2008, consulté le 07 juillet 2022. URL : http://journals.openedition.org/ ema/1240 ; DOI : https://doi.org/10.4000/ema.1240

\section{Ce document a été généré automatiquement le 7 juillet 2022}

Tous droits réservés 


\title{
Les spécificités de l'économie égyptienne
}

\author{
Bruno Cabrillac
}

\section{NOTE DE L'ÉDITEUR}

Bruno Cabrillac est économiste à la Banque de France et ex-attaché financier du ministère français des Finances pour le Proche et le Moyen-Orient.

1 Le thème traité ci-après est sans doute quelque peu paradoxal. La notion de spécificité est en effet suspecte aux yeux des économistes qui prétendent à l'universalité de leurs modèles, voire d'un modèle. Dès lors, l'analyse des spécificités ressortit davantage à la géographie économique, voire à l'histoire ou à la sociologie. Elle conduit aussi à énoncer des lieux communs, les spécificités étant des signes distinctifs et pouvant, comme tels, être perçus de tous. Mais il n'est pas toujours inutile de rappeler les lieux communs : on en recherche rarement les raisons ou les fondements. Cependant, après avoir développé les spécificités de l'économie égyptienne, je reviendrai à la macroéconomie en examinant comment l'ajustement structurel qu'applique aujourd'hui l'Égypte s'inscrit dans les spécificités de son économie.

2 Cette étude, disons-le en préambule, ne prétend pas à l'exhaustivité. Sans doute y a-t-il des spécificités propres à la société égyptienne mais, n'étant pas sociologue, je ne suis pas compétent pour les identifier et tenter d'en mesurer les conséquences sur l'économie.

Il y aussi des spécificités que je souhaite évoquer sans m'y appesantir. Certaines tiennent à l'appartenance de l'Égypte à des ensembles plus larges :

4 1) L'Égypte est un pays en voie de développement à revenu intermédiaire. Les principaux indicateurs socio-économiques témoignent de l'appartenance de l'Égypte à ce groupe. 
Espérance de vie

Taux de natalité

Taux de mortalité

Habitants par médecin

Habitants par lit d'hópital

Accès à l'électricité

\begin{tabular}{ll}
\multicolumn{1}{c}{ milieu rural } & $19 \%$ \\
milieu urbain & $77 \%$ \\
Acces à l'eau potable & $90 \%$ \\
Part de l'agriculture dans l'emploi & $46 \%$ \\
Taux de scolarisation & \\
\multicolumn{1}{c}{ primaire } & $90 \%$ \\
secondaire & $69 \%$
\end{tabular}

63 ans

33 p. 1.000

9 p. 1.000

767.

480

$19 \%$

$77 \%$

$90 \%$

$46 \%$

$69 \%$
l'Égypte a de meilleurs indicateurs sociaux sur plusieurs points :

1. un système de santé plus développé, au moins quantitativement ;

2. une ration alimentaire moyenne élevée, proche de celle des pays développés;

3. une espérance de vie élevée, peut-être conséquence des deux points précédents ;

4. un accès presque général à l'eau potable, la quasi-totalité de la population vivant à proximité du Nil.

2) L'Égypte est un pays arabe et, en tant que tel, s'inscrit dans un ensemble économique original. Je me contenterai d'en souligner les principaux traits : c'est un ensemble dans lequel il y a très peu d'échanges de biens et beaucoup de mouvement de personnes. Le premier trait est caractéristique des pays du Sud, les échanges Nord-Sud y étant prépondérants. L'Égypte n'échappe pas à ce schéma : les importations égyptiennes en provenance du Moyen-Orient arabe ne représentent qu'environ $1 \% \mathrm{du}$ total des importations, et les exportations vers le Moyen-Orient moins de $5 \%$ des exportations totales - y compris les exportations de pétrole égyptien vers Israël, prévues par les accords de Camp David. Le deuxième trait - l'intense circulation des hommes - est plus spécifique du Monde arabe. Il est dû à une grande unité culturelle - même langue, même religion dominante, fonds culturel commun -, à une disparité importante des revenus mais aussi des équipements culturels. L'Égypte est un élément central des migrations au sein du Monde arabe puisqu'elle en est à la fois le phare culturel et le principal réservoir de travailleurs.

7 3) Enfin, l'Égypte est un pays méditerranéen et, comme tel, paradoxalement peu tourné vers la mer. Le déclin pluriséculaire de la Méditerranée comme lieu d'échange trouve à Alexandrie son illustration la plus frappante. Pays, donc, peu tourné vers la mer, avec une activité de pêche réduite, mais qui s'inscrit néanmoins dans l'ensemble méditerranéen : l'Europe, la Turquie et le Maghreb représentent $55 \%$ à $60 \%$ de ses 
échanges commerciaux, les touristes européens constituent la grande majorité de ses visiteurs. En outre, le tropisme de la diplomatie égyptienne vers le monde arabe s'atténue au profit d'une politique méditerranéenne active - par exemple, dans les conférences « $5+5$ » ou comme partenaire de la « politique méditerranéenne rénovée » de la CEE - qui souligne son appartenance au monde méditerranéen.

Laissant de côté ces spécificités tenant à l'appartenance de l'Égypte à des ensembles plus vastes, je traiterai plus à fond deux caractéristiques de l'économie égyptienne : la rareté de l'eau et de l'espace, d'une part, l'importance de la rente d'autre part.

\section{La rareté de l'eau et de l'espace}

9 Rareté de l'eau tout d'abord: l'Égypte se trouve dans une zone désertique et le Nil constitue la quasi-totalité de ses ressources hydrauliques. De plus, aux termes de l'accord signé avec le Soudan en 1959 sur le partage des eaux du Nil, l'Égypte ne peut disposer que de $75 \%$ du débit moyen du fleuve, soit 56 milliards de $\mathrm{m}^{3}$ environ par $\mathrm{an}^{1}$. Rareté de l'espace ensuite: la surface agricole utile n'est que de $50.000 \mathrm{~km}^{2}$, soit environ $4 \%$ du territoire, et $95 \%$ des habitants ( 57 millions d'âmes) sont concentrés le long du Nil sur moins de $5 \%$ du territoire, c'est-à-dire une surface équivalente à la Hollande.

10 Certes, d'autres économies dans le monde souffrent d'une rareté de l'eau et/ou de l'espace, mais rarement autant qu'en Égypte. Les deux contraintes sont liées dans la mesure ou l'espace n'est limité que par la rareté de l'eau et la dépendance à l'égard du Nil.

11 Mesurer le degré de rareté de l'eau en Égypte n'est pas chose facile. D'une part, le débit du Nil à l'embouchure, qui mesure les quantités d'eau utilisables, n'est pas précisément connu. D'autre part l'estimation, par les spécialistes, des marges de capacité disponibles, varie dans une large proportion : de $15 \%$ à $40 \%$ selon les estimations. Si la régularité des ressources en eau pallie dans une certaine mesure leur rareté, surtout depuis le nouveau barrage d'Assouan, elles sont cependant- autre caractéristique des ressources en eau - géopolitiquement menacées, les sources et le cours supérieur du Nil se trouvant dans une région - Ethiopie, Kenya, Soudan - particulièrement instable. Et les efforts séculaires de l'Égypte pour contrôler indirectement les eaux du Nil ont échoué. Cette rareté de l'eau, je l'ai mentionné plus haut, implique une forte rareté de l'espace. Rareté de l'espace agricole d'abord: depuis le début des années 60, 800.000 feddans environ ${ }^{2}$, soit 6 à $7 \%$ de la surface agricole utile, ont été mangés par l'urbanisation; il est vrai que dans le même temps, 1,5 million de feddans ont été mangés sur le désert par la bonification des terres.

12 Le desserrement réel de la contrainte spatiale est donc limité - 5 à $6 \%$ de la surface agricole utile en trente ans et la tendance est plutôt au resserrement de la contrainte. En effet, l'urbanisation se poursuit alors que la bonification des terres désertiques, coûteuse en eau, trouvera vite ses limites.

13 Conséquence de la rareté de l'espace agricole, la progression de la production agricole ne peut venir que des gains de productivité. Or ceux-ci ont été très faibles depuis les années 60, pour trois raisons: si l'usage des engrais a permis d'améliorer la productivité, le barrage d'Assouan empêche une partie du limon, autrefois transporté par le Nil, de se déposer dans la plaine fluviale, ce qui doit être compensé par l'usage 
accru d'engrais. Ensuite, les terres gagnées sur le désert ont un rendement inférieur à celui des terres conquises par l'urbanisation. Enfin, la productivité du travail n'a guère progressé, essentiellement en raison du ralentissement de l'exode rural ralentissement plus accentué qu'ailleurs, sans doute parce que la vallée du Nil est un continuum urbain.

La faible augmentation de la production agricole depuis les années 60 , jointe à une croissance démographique rapide, a entrâné, depuis le milieu des années 70 , le déficit des échanges agricoles. Il convient de distinguer l'autosuffisance alimentaire - que l'Égypte n'a jamais réellement atteinte depuis le début du siècle - et l'excédent des échanges agricoles, qui était généré par les exportations de coton. Cette distinction est intéressante pour apprécier la politique agricole au regard des contraintes naturelles: l'autosuffisance alimentaire, au contraire du rééquilibrage des échanges agroalimentaires, n'est pas souhaitable. En effet, d'une part, le développement de la production céréalière se fera au détriment de cultures intensives, à plus forte valeur ajoutée par hectare, ou de cultures faiblement consommatrices d'eau, cultures plus conformes aux avantages comparatifs de l'Égypte. D'autre part, l'Égypte peut profiter du fait qu'elle est un acheteur important sur le marché céréalier, structurellement excédentaire, pour obtenir de meilleurs prix : stratégie qu'elle a menée avec succès auprès de l'Australie, des États-Unis et de la France.

15 Autre conséquence de la rareté de l'eau et de l'espace : la dramatisation du problème démographique. Cette dramatisation est ancienne ${ }^{3}$. Or, la démographie égyptienne n'est pas plus expansive que celle des autres pays arabes ou de la majorité des pays africains. Le taux de natalité a baissé en Égypte plus vite que dans d'autres pays du Tiers-Monde et, en dépit d'une certaine remontée, le taux de croissance démographique - qu'il est difficile de connaître précisément mais qui est probablement compris entre 2,1 et 2,4\% - est très inférieur à celui du Yémen, des pays de la Péninsule arabique, de la Syrie ou des Territoires occupés. L'Égypte est donc un pays de basse pression démographique au Moyen-Orient, et c'est pourtant l'endroit où la croissance démographique est la plus dramatisée, conséquence de la rareté de l'eau et de l'espace.

Autre domaine dans lequel cette double rareté a des conséquences importantes: l'urbanisation et l'environnement. Le développement urbain en Égypte est marqué par la rareté de l'espace: absence de rupture entre ville et campagne, acceptation d'une extrême centralisation. Je n'insisterai pas sur ce point car nous sommes aux marges de l'économie. L'acuité des problèmes d'environnement et de leur perception sera sans doute, dans le futur, la conséquence la plus évidente de la rareté de l'eau et de l'espace.

\section{Une économie de rente}

17 Deuxième caractéristique : l'économie égyptienne est une économie de rente et même une économie de rentiers. J'utiliserai ici le terme de « rente » dans son sens commun et non dans son sens ricardien (schématiquement, une activité liée à une ou des caractéristiques structurelles), c'est-à-dire comme un revenu non lié à une activité productive interne.

18 On peut identifier les revenus de rente dans la balance des paiements. 
Il y a d'abord les « rentes géographiques » qui viennent du fait que l'Égypte est un pays entre deux mers - la Mer Rouge et la Méditerranée. Les plus importantes de ces rentes sont :

1. le Canal de Suez, qui est devenu la troisième source de recettes extérieures de l'Égypte, avec plus de 2 milliards de dollars en-1991;

2. l'oléoduc Sumed, qui double le Canal de Suez et permet de faire transiter des produits pétroliers entre Suez et Port Saïd, et dont les redevances représentent 200 millions de dollars par an.

Deuxième type de rente, la rente pétrolière. Aux exportations de pétrole, il faut ajouter les investissements des compagnies pétrolières qui travaillent en Égypte, dans la mesure où ils profitent aux entreprises locales, et les boni versés pour l'acquisition des droits de concession. En fait, la balance des paiements ne donne qu'une idée partielle de la rente pétrolière puisque celle-ci concerne l'ensemble de la production, y compris ce qui est utilisé pour la consommation domestique.

21 Troisième type de rente extérieure, ce sont les revenus des facteurs de production utilisés à l'extérieur de l'Égypte. Les transferts des travailleurs émigrés sont la première source de revenus extérieurs de l'Égypte et ont rapporté 5,2 milliards de dollars en 1991-92 (année fiscale débutant au 1er juillet). Parallèlement, les revenus d'investissement, c'est-à-dire de tous les placements extérieurs des résidents égyptiens, ont atteint 1,5 milliard de dollars la même année.

Quatrième type de rente dont bénéficie l'Égypte, la rente géopolitique. Sans débattre ici du pourquoi ou du comment l'Égypte a pu bénéficier d'une rente géopolitique et l'exploiter, je soulignerai simplement l'ampleur des revenus qu'elle en tire. Il y a d'abord les dons américains, conséquence des accords de Camp David, qui représentent 3 milliards de dollars par an, dont 1,8 milliard de dollars d'aide militaire, auxquels s'est ajoutée en 1991 l'annulation de la dette militaire (7 milliards de dollars). Il y a ensuite les dons des pays du Golfe, un temps interrompus mais qui ont repris depuis quelques années. L'Égypte est historiquement la première bénéficiaire de l'aide arabe.

Il est difficile de connaitre exactement le montant des dons des monarchies du Golfe en raison de la confidentialité de ce type d'opération. Depuis la guerre contre l'Irak, les dons en provenance des pays du Golfe sont considérables, sans doute plus de 8 milliards de dollars, y compris les remises de dette. Il y a enfin l'ensemble de l'aide occidentale, hors États-Unis, particulièrement importante depuis le début des années 80. L'abandon en 1991, par les créanciers du Club de Paris, de la moitié de leurs créances est une bonne illustration des revenus de la rente géopolitique, puisqu'il est explicitement souligné dans les termes de l'accord que la faveur consentie à l'Égypte est due à son rôle dans la guerre du Golfe.

24 À titre d'exemple, le tableau ci-après montre l'importance de ces quatre types de rente dans la balance des paiements égyptienne entre 1988/89 et 1990/91, année il est vrai exceptionnelle. Si, chiffrés par la balance des paiements, les revenus des rentes sont considérables, ils n'en demeurent pas moins sous-estimés, au moins pour trois postes : 
Répartition des 4 types de rentes dans la balance des paiements égyptienne entre 1988/89 et 1990/91 (en milliards de \$ US)

\begin{tabular}{|l|c|c|c|}
\hline & $1988 / 89$ & $1989 / 90$ & $1990 / 91$ \\
\hline Canal de Suez - Summed & 1,5 & 1,7 & 1,9 \\
Exportations de pétrole & 1,2 & 1,2 & 2,0 \\
Revenus des facteurs * & 4,5 & 4,9 & 5,2 \\
Aide extérieure & 0,8 & 1,0 & 4,8 \\
Total & 8,0 & 8,8 & 13,9 \\
& & & \\
p.m. total des recettes & & & \\
extérieures courantes & 12,4 & 14 & 19,2 \\
p.m. remise de dettes & - & 7,7 & - \\
\hline
\end{tabular}

- travail et capital

1. les revenus d'investissement à l'extérieur, dans la mesure où l'on estime les capitaux des résidents égyptiens placés à l'étranger entre 20 milliards de dollars (chiffres de la Banque des règlements internationaux) et 40 milliards de dollars ;

2. les transferts de travailleurs émigrés, dont une partie n'emprunte pas les canaux officiels ;

3. l'aide arabe, dont la majeure partie n'est pas officiellement comptabilisée.

Si les revenus de rente sont, en Égypte, remarquables par leur ampleur, ils le sont aussi par la diversité des sources, puisqu'il y a au moins quatre facteurs principaux de rente et qu'on pourrait, un peu extensivement il est vrai, en identifier un cinquième, de type "historique" : l'Égypte est en effet redevable à son ancienne civilisation d'une grande partie de son tourisme.

L'économie de rente égyptienne présente deux autres caractéristiques.

Tout d'abord, la confiscation - ou la tentative de confiscation - de la rente par l'État: nationalisation du Canal de Suez et de la production de pétrole, institution d'une taxe sur les salaires des travailleurs émigrés. La surévaluation systématique du cours officiel de la livre égyptienne a longtemps constitué un moyen indirect de confiscation de la rente. Mais, à l'inverse de ce qui s'est passé dans d'autres pays pétroliers, l'État n'a pu en confisquer qu'une partie. Les transferts de travailleurs émigrés ou les revenus d'investissement sont très sensibles aux mesures fiscales ou de contrôle des changes. La confiscation de ces revenus par l'État est particulièrement difficile.

Ensuite, le mode de redistribution de la rente, qui s'est fait essentiellement au profit des consommateurs par un mécanisme de prix subventionnés. Les rentes extérieures confisquées par l'État ont ainsi été utilisées pour maintenir les produits de base à des prix artificiellement bas. Le mécanisme de distribution n'a d'ailleurs pas été réservé aux rentes extérieures. Les rentes de monopole - il y a de nombreux monopoles publics en Égypte - et la rente foncière ont été largement redistribuées aux consommateurs par la généralisation de la subvention aux prix à la consommation, allant parfois audelà de la redistribution de la rente puisque certains monopoles ne couvrent pas leur coûts.

Les conséquences de ce système sont nombreuses : 
30 échelle des prix est faussée, ce qui entraîne une allocation défectueuse des ressources. L'exemple le plus flagrant en est l'utilisation du pain dans l'alimentation animale alors que l'essentiel des besoins en blé est importé ;

31 - le fait de subventionner les produits de base permet de maintenir les bas salaires à des niveaux très faibles, puisque le revenu de subsistance est lui-même faible ;

32 - la distribution de la rente par des prix subventionnés profite aux pauvres comme aux riches, puisque ces derniers sont aussi consommateurs et qu'elle a un effet dépressif sur les bas salaires. L'effet redistributif est donc limité. Les disparités de richesse se sont sensiblement accrues ces dernières années. Selon une étude effectuée pour la Banque mondiale en 1986, les $10 \%$ les plus riches recevaient $55 \%$ du revenu national, les $20 \%$ les plus pauvres en percevant à peine $3 \%$. Cette structure des revenus était alors une des plus inégalitaires de la région ;

33 prix à la consommation et non par celui des salaires, l'Égypte a évité ce qu'on appelle le "mal hollandais », phénomène bien connu des économistes et qui s'est produit par exemple pour les Pays-Bas au moment où ce pays a exploité une importante rente gazière : le secteur qui bénéficie de la rente peut la confisquer au profit de ses salariés. Les augmentations de salaires s'étendent ensuite aux autres secteurs qui, ne bénéficiant pas de rente, perdent de leur compétitivité et doivent réduire leur activité. Ce phénomène a totalement épargné l'Égypte.

Telles sont donc, dans leurs grandes lignes, les deux spécificités essentielles de l'économie égyptienne. Examinons pour conclure quelles sont les interactions entre la politique d'ajustement en cours, qui s'inspire d'un modèle général, et ces spécificités.

Premier point: la dépendance alimentaire, conséquence de la rareté de l'eau et de l'espace, fait de l'ajustement, en cas de crise des paiements, une nécessité absolue, voire dramatique. On l'a un peu oublié dans la mesure où l'Égypte vit aujourd'hui dans une certaine aisance financière. Mais au début de 1990, avant la crise du Golfe, la crise des paiements était aiguë et le problème fondamental des autorités égyptiennes était de remplir les silos. L'ajustement extérieur, c'est-à-dire la réduction des importations autres que celles des produits de première nécessité pour pouvoir acheter du blé, était incontournable.

xième point: l'ajustement tel qu'il est pratiqué cherche à remettre en cause le processus de distribution de la rente en supprimant les subventions, en rétablissant la vérité des prix et en substituant aux subventions une aide aux personnes. Processus difficile et douloureux parce qu'il touche aux structures mêmes de la société.

Troisième point : le succès de l'ajustement repose sur l'élasticité des revenus extérieurs pour que, dans un deuxième temps, le développement des exportations se substitue à la contraction des importations de façon à rééquilibrer les comptes extérieurs. Or, une grande partie des ressources extérieures de l'Égypte provient de revenus de rente, par nature inélastiques aux données macro-économiques intérieures, ce qui rend plus aléatoire et plus longue à réaliser, sans doute, la politique d'ajustement.

Ce sont donc, en partie, les spécificités de l'économie égyptienne qui expliquent les difficultés, plus grandes qu'ailleurs, de mise en place de la politique d'ajustement en Égypte. 


\section{NOTES}

1. Voir à ce sujet Égypte/Monde arabe $\mathrm{n}^{\circ} 10$ (éléments d'analyse et dossier de presse), NDLR.

2. 1 feddan $=0,42$ ha, NDLR.

3. Dans Le Tarbouche, le roman de Robert Solé, un des personnages est en permanence obsédé par l'explosion démographique, cela dès les années 20 .

INDEX

Mots-clés : économie, rente 